\title{
Future directions of biosensors
}

\section{Editorial}

When we compare the modern sophisticated biosensors with the natural one (for example, nose) we understand the presently developed biosensor is still crude and simplistic. ${ }^{1}$ The necessity for the real-time, easy to handle and highly sensitive detection devices for both health (e.g. clinical diagnostics) and non-health applications (e.g. veterinary research, agriculture, industrial processes and pollution control) has led to an ever increasing demand for biosensors improvement.$^{1,2}$

Biosensors are receiving significant attention today for various reasons. One of them is the reduction of the cost of computers and microprocessors that contributed a great demand for distributed intelligence that requires monitoring for better control. ${ }^{1,3}$ This demand has resulted in a greater requirement of the robust and timely manner for the development of the biosensors to provide as the front end of measurement and control systems. ${ }^{4}$

Naturally the improvement of the biosensor is always ongoing. Increasing attention in the area of engineering has to be in both the basic components as well as the biosensor as a whole device. Technological advancements of the biosensors will accelerate the applications of intelligent instrumentation, micro and nanoelectronics and methods of multivariate signal processing, for example chemometrics and artificial neural networks. ${ }^{1,2,4,5}$

A microarray biosensor strategy, adaptable to multiple analyte detection will allow spreading of the biosensor technology and reduce the developmental costs over several competitive products with the presently available instruments and will allow them to operate under field conditions. ${ }^{1,5,6}$

Future directions in biosensors technology will address the increased range of analytes, completely integrated systems possessing various phases of sample handling, microfluidics, detection and display possibly telemetry, renewed emphasis on whole cell and tissue biosensors. ${ }^{1,5-7}$ It will direct us toward use of embedded systems, telecommunications capability, wearable biosensors and the biosensor devices that can be mass produced and used in a variety of applications including homes, hospitals, automobiles, toxic dump sites as well as many other applications..$^{1,5,7}$

The new trends are the development of a biosensor method that would carried out in volume not on surface, using nanotechnology such as quantum dot nanoparticles, nanowires and nanotubes, development of artificial antibodies such as aptamer or peptides selected by phage display methods and development of lab-on-a-chip for the detection of the antigens of the specific target the areas. ${ }^{1,2,3,8-10}$ Few of the newest application area of biosensor are biodiesel quality sensing and electrochemical based biosensor, multi-dimensional pain biosensors. ${ }^{2,8,10}$

Hopefully, biosensors can eventually be used in vivo in order to get real time measurements of molecular interactions within the body in the near future. ${ }^{1,10}$ The multidisciplinary arena of expertise necessary for the development of biosensors can be sustained by the

\author{
Md Zakir Hossain,' Rozina Akter, ${ }^{2}$ Anita L \\ Jackson,' Dev S Shrestha ${ }^{3}$ \\ 'Department of Pharmaceutical Sciences, North Carolina \\ Central University, USA \\ ${ }^{2}$ BioMedNanoTech, Inc., USA \\ ${ }^{3}$ University of Idaho, USA
}

Correspondence: Md Zakir Hossain, Assistant Research Professor, Department of Pharmaceutical Sciences, Biomanufacturing Research Institute and Technology Enterprise (BRITE), North Carolina Central University, BRITE Building, Room \# 1013, 180 I Fayetteville Street, Durham, NC 27707, Tel 919530 6884, Fax 9195306894 Email zhossain@.ccu.edu

Received: January 20, 2016 | Published: January 29, 2016

collaboration of many areas of academia and industry. ${ }^{1}$ Though the result is a slow process but most probably the only realistic path to successful future advances in biosensor technology.

\section{Acknowledgments}

None.

\section{Conflicts of interest}

None.

\section{References}

1. Hossain MZ Biosensors applications in biological and agricultural engineering and design of a novel potentiometric lipase biosensor for biodiesel quality sensing. M. Engr. Design project thesis, University of Idaho, USA. 2008

2. Hossain MZ, Shrestha DS, Kleve MG Biosensors for Biodiesel Quality Sensing. Journal of the Arkansas Academy of Science. 2010;64:80-85.

3. Wanekaya AK, Chen W, Nosang VM et al. Nanowire-Based Electrochemical Biosensors. Electroanalysis 2006;18(6):533-550.

4. Gooding JJ, Yang W, Hibbert DB "Biomimetic sensors for the detection of metal ions." Chemistry in Australia. 2012;69(1):8-10.

5. Ozkan-Ariksoysal D Biosensors and their application in healthcare: hot topics. Biosensors 2-4. 2013

6. Mohanty SP, Kougianos E "Biosensors: A tutorial review". Potentials IEEE. 2006;25(2):35-40.

7. Wang J Nanomaterial-based electrochemical biosensors. Analyst. 2005;130(4):421-426.

8. Omole MA, Noah N, Zhou L et al. Spectroelectrochemical characterization of pain biomarkers. Anal Biochem. 2009;395(1):54-60.

9. Erdem A, Ozsoż M Electrochemical DNA biosensors based on DNAdrug interactionś. Electroanalysis. 2002;14(14):965-974.

10. Gooding JJ, Liu G Electrochemical sensor. US Patent 8,110,079. 2012. 